\title{
AVALIAÇÃO DE ADSORVENTES COMERCIAIS PARA A REMOÇÃ̃O DE COMPOSTOS DE ENXOFRE E NITROGÊNIO PRESENTES NO ÓLEO DIESEL COMERCIAL
}

\author{
F. A. V. PEREIRA ${ }^{1}$, C. I. YAMAMOTO ${ }^{3}$, S. S. X. CHIARO ${ }^{4}$, S. M. A. GUELLI U. de SOUZA ${ }^{2}$, \\ A. A. ULSON DE SOUZA ${ }^{5}$ \\ 1,2,5 Universidade Federal de Santa Catarina, Departamento de Engenharia Química e Engenharia de \\ Alimentos \\ ${ }^{3}$ Universidade Federal do Paraná, Departamento de Engenharia Química \\ ${ }^{4}$ PDAB/HPE - CENPES/PETROBRAS. \\ E-mails para contato: fulvy.antonella@hotmail.com, selene@enq.ufsc.br, augusto@enq.ufsc.br
}

\begin{abstract}
RESUMO - Com o processamento de petróleos mais pesados, a hidrodessulfurização (HDS) passou por modificações que aumentaram a severidade das condições operacionais e com isso, e com isso reduziram-se as cargas de óleo diesel a um custo mais elevado. $\mathrm{O}$ presente estudo avaliou alguns adsorventes comerciais como resina, argila e carvão ativado para remoção adsortiva de enxofre e nitrogênio presentes no óleo diesel comercial, como um processo complementar à HDS. Para o carvão ativado, que apresentou melhores resultados, foi realizado o estudo cinético e verificou-se que o tempo de $4 \mathrm{~h}$ de contato foi economicamente favorável e que os dados foram melhor ajustados pelo modelo de pseudo-segunda ordem de Lagergren. Avaliou-se que o carvão ativado estudado pode remover em torno de $46,0 \%$ do enxofre total e $90,0 \%$ do nitrogênio total inicialmente presentes no óleo diesel comercial. Tal fato apresenta a adsorção com carvão ativado como um processo eficaz na dessulfurização e desnitrogenação profunda do óleo diesel.
\end{abstract}

\section{INTRODUÇÃO}

As restrições referentes à concentração de enxofre em combustíveis aplicados ao transporte estão cada vez mais severas nas últimas décadas, sendo que em alguns países limita-se a poucas partes por milhão (Gutiérrez e Klimova, 2011; Blanco-Brieva et al., 2011). No Brasil, segundo a Resolução da ANP N 65 (2011) a partir de $1^{\circ}$ de janeiro de 2013, o óleo diesel S50 (50 ppm de enxofre) será substituído, integralmente, pelo óleo diesel S10 (10 ppm de enxofre).

Atualmente, o processo mais utilizado para remoção de enxofre nas refinarias é a Hidrodessulfurização (HDS), método tradicional que remove enxofre dos combustíveis por meio de reação catalítica com hidrogênio a elevadas temperaturas $\left(300-340{ }^{\circ} \mathrm{C}\right)$ e elevadas pressões $(20$ a 100 atm de $\mathrm{H}_{2}$ ) (Yang et al., 2013). Todavia, nestas condições de operação a HDS é eficiente na 
remoção de tióis, sulfetos e dissulfetos, entretanto, não reduz tiofenos, benzotiofenos e benzotiofenos substituídos, pois ocorre a estabilização aromática de seus anéis (Kumar et al., 2012). Portanto, para alcançar os desafios da produção com teor de enxofre menor que 10 ppm são necessários avanços no desenvolvimento do processo com altos investimentos, além do custo operacional ser elevado, devido ao aumento na severidade das condições operacionais (Fallah e Azizian, 2012; Paz-Zavala et al., 2013). Devido a estas dificuldades novas tecnologias para ultradessulfurização estão sendo estudadas, entre elas destaca-se a dessulfurização por adsorção.

O processo adsortivo tem atraído atenção na dessulfurização de combustíveis líquidos devido ao baixo consumo de energia, operação a temperatura e pressão ambiente, não utilização de hidrogênio e viabilidade de regeneração do adsorvente (Tanabe et al., 2011). Dentre os vários adsorventes aplicados o carvão ativado tem-se destacado por apresentar elevada área superficial e distribuição de poros, além da possibilidade de regeneração (Al-Ghouti et al., 2010). Outros materiais são muito empregados no processo de adsorção de compostos orgânicos presentes em combustíveis como as resinas e argilas. Algumas resinas poliméricas, copolímeros não-iônicos de estireno e divinilbenzeno, líquidos iônicos clorados foram utilizados na remoção de enxofre e nitrogênio presentes no óleo diesel (Xie et al., 2010, Guo et al., 2010).

\section{MATERIAIS E MÉTODOS}

\section{1 Óleo Diesel Comercial}

O óleo diesel utilizado na pesquisa foi obtido da Refinaria Getúlio Vargas (REPAR), no Paraná. A amostra utilizada é produto da HDS e não teve nenhuma modificação com aditivos e/ou biodiesel. Realizou-se análise físico-química da amostra de diesel utilizada na pesquisa, e foram obtidas as propriedades apresentadas na Tabela 1.

Tabela 1 - Propriedades físico-químicas da amostra de óleo diesel avaliada.

\begin{tabular}{|c|c|c|c|c|c|c|}
\hline Parâmetros & $\begin{array}{l}\text { Ponto de Fulgor }^{\mathrm{a}} \\
\left({ }^{\circ} \mathrm{C}\right)\end{array}$ & $\begin{array}{l}\text { Enxofre Total }^{\mathrm{b}} \\
(\mathrm{mg} / \mathrm{Kg} \mathrm{S})\end{array}$ & $\begin{array}{l}\text { NitrogênioTotal } \\
(\mathrm{mg} / \mathrm{Kg} \mathrm{N})\end{array}$ & $\begin{array}{l}\text { Massa }_{\text {específica }^{\mathrm{d}}} \\
\left(\mathrm{Kg} / \mathrm{m}^{3}\right)\end{array}$ & $\begin{array}{l}\text { Viscosidade }^{\mathrm{e}} \\
\mathrm{a} 40^{\circ} \mathrm{C}\end{array}$ & $\begin{array}{l}\text { Viscosidade }^{\mathrm{e}} \\
\mathrm{a} 70^{\circ} \mathrm{C}\end{array}$ \\
\hline S250 & 92,0 & 235 & 228 & 841,1 & 3,3212 & 1,9359 \\
\hline
\end{tabular}

a: Realizado no equipamento PENSKY PMA4; b: Método ASTM D4294; c: Método ASTM D4629; d: Método ASTM D4052; e: Método ASTM D445.

\subsection{Adsorventes avaliados}

Resinas: As resinas utilizadas no presente estudo são da empresa Mitsubishi Chemical classificação Diaion \& Sepabeads (Synthetic Adsorbents): HP 20, SP 70 e SP 700. Sendo as resinas HP20, SP70 e SP700, resinas do tipo aromáticas e copoliméricas de divinilbenzeno-estireno, não substituídas, ou seja, sem grupos funcionais. Dentre essas resinas aromáticas não substituídas a HP20 não passou por nenhum tratamento adicional industrialmente, sendo também classificada como área superficial 
normal, já as resinas SP70 e SP700 passaram por tratamentos especiais (não especificados pelo fornecedor), apresentando áreas superficiais mais elevadas.

Argila: Argila montmorilonita organofílica Claytone 40 (fabricado pelo Buntech e cedido pela Bentonite União Nordeste).

Carvão Ativado: O carvão ativado empregado neste estudo é proveniente da indústria paranaense Fábrica Brasileira de Catalisadores (FBC), sendo o precursor do adsorvente a casca do coco de babaçu. $\mathrm{O}$ adsorvente passou por ativação física e não teve nenhum tratamento adicional para sua utilização, apenas secagem para remoção da umidade e gases. Para o estudo a amostra foi denominada CA.

\subsection{Avaliação da Capacidade de Remoção dos Compostos de Enxofre e Nitrogênio}

O procedimento experimental de adsorção em batelada foi realizado na Incubadora Tipo Shaker Orbital para todos os adsorventes avaliados. Primeiramente ambientou-se o óleo diesel durante $1 \mathrm{~h}$ à temperatura de $40{ }^{\circ} \mathrm{C}$, mesma temperatura utilizada para o ensaio, e o adsorvente foi seco em estufa a $130{ }^{\circ} \mathrm{C}$ por $12 \mathrm{~h}$ e mantido em dessecador até o uso. Aproximadamente 2,0 g de adsorvente foram colocados em contato com $20 \mathrm{~mL}$ do óleo diesel previamente aquecido, a mistura foi então levada ao Shaker Orbital à temperatura de $40^{\circ} \mathrm{C}$ e agitação de $150 \mathrm{rpm}$, durante $24 \mathrm{~h}$, realizou-se a amostragem e o conteúdo foi mantido refrigerado para posterior leitura no analisador de enxofre e nitrogênio.

\subsection{Determinação de Enxofre e Nitrogênio Totais}

A determinação do teor de enxofre e nitrogênio totais residuais no óleo diesel foi realizada por meio do analisador de enxofre Elementar Trace SN cube. As análises foram efetuadas em amostras líquidas, para as quais o método de análise se resume na injeção em um tubo catalítico de combustão de amostras, separação de gases estranhos e detecção por meio de Fluorescência UV. A concentração da fase adsorvida (mg S/g CA) foi calculada por balanço de massa, de acordo com a Equação 1,

$$
q=\frac{\left(C_{i}-C_{f}\right) \cdot V_{s o l} \cdot \rho_{s o l}}{m_{a d s}}
$$

onde, $C_{i}$ é a concentração inicial de enxofre ou nitrogênio na solução em $\mathrm{ppm}(\mathrm{m} / \mathrm{m}), \mathrm{C}_{\mathrm{f}}$ é a concentração final de enxofre na solução em ppm $(\mathrm{m} / \mathrm{m}), \mathrm{V}_{\text {sol }}$ é o volume da solução utilizada $(\mathrm{mL})$, $\rho_{\text {sol }}$ é a massa específica da solução $(\mathrm{g} / \mathrm{mL})$ e $\mathrm{m}_{\mathrm{ads}}$ é a massa de adsorvente utilizada $(\mathrm{g})$.

\subsection{Cinética de Adsorção}

A fim de compreender melhor o processo de adsorção dos compostos de enxofre e nitrogênio modelos cinéticos comumente utilizados, como o medelo de Lagergren de pseudo-primeira ordem e pseudo-segunda ordem foram avaliados para melhor descrever a cinética de adsorção dos compostos 
envolvidos pelo carvão ativado.

O procedimento foi executado colocando-se 4,0 g de carvão ativado em contato com $20,0 \mathrm{~mL}$ de óleo diesel, as amostragens foram realizadas durante intervalos de tempo de 1 minuto a 120 horas.

\section{RESULTADOS E DISCUSSÃO}

\subsection{Avaliação da Capacidade de Remoção dos Compostos de Enxofre e Nitrogênio}

Os resultados referentes à remoção de compostos de enxofre e nitrogênio provenientes do óleo diesel comercial para todos os adsorventes testados serão apresentados a seguir. O óleo diesel comercial apresentou concentração inicial de $235 \mathrm{ppm}(\mathrm{m} / \mathrm{m})$ de enxofre e $228 \mathrm{ppm}(\mathrm{m} / \mathrm{m})$ de nitrogênio.

Resinas: A remoção dos compostos sulfurados e nitrogenados obtida para cada resina avaliada á apresentada na Tabela 2.

Tabela 2 - Resultados obtidos para as resinas avaliadas.

\begin{tabular}{|c|c|c|c|c|}
\hline Adsorventes & $\begin{array}{c}\mathrm{q} \mathrm{S} \\
(\mathrm{mg} \mathrm{S} / \mathrm{g})\end{array}$ & $\begin{array}{c}\% \mathrm{~S} \\
\text { Removida }\end{array}$ & $\begin{array}{c}\mathrm{q} \mathrm{N} \\
(\mathrm{mg} \mathrm{N} / \mathrm{g})\end{array}$ & $\begin{array}{c}\% \mathrm{~N} \\
\text { Removida }\end{array}$ \\
\hline HP 20 & 0,2 & 11,4 & 0,6 & 28,9 \\
\hline SP 70 & 0,3 & 16,3 & 0,8 & 40,0 \\
\hline SP 700 & 0,4 & 19,6 & 1,0 & 54,7 \\
\hline
\end{tabular}

A adsorção com as resinas apresentou-se mais favorável para os compostos nitrogenados, sendo que a resina SP700, foi a que apresentou maior remoção de compostos de enxofre (19,6\%) e de nitrogenados (54,7\%). Provavelmente o melhor resultado para a SP700 deve-se a sua maior área superficial em relação às demais, sendo que as áreas são $600 \mathrm{~m}^{2} / \mathrm{g}, 800 \mathrm{~m}^{2} / \mathrm{g}$ e $1200 \mathrm{~m}^{2} / \mathrm{g}$, para as amostras HP20, SP70 e SP700, respectivamente. O ganho na capacidade adsortiva seguiu o aumento na área superficial, fato que confirmou as expectativas, uma vez que estas resinas não são substituídas, sendo assim, sua adsorção é praticamente física e a porcentagem de remoção é proporcional à área disponível para a adsorção.

No que se refere a maior adsorção de compostos nitrogenados, tal fato foi observado por estudos de Xie et al. (2009 e 2010), no qual adsorções foram realizadas em combustíveis sintéticos com dibenzotiofeno representando os compostos de enxofre e carbazol os compostos de nitrogênio. Segundo Koriakin et al. (2010) maior seletividade foi observada para compostos nitrogenados, possivelmente por tais compostos terem preferência na adsorção aos compostos sulfurados, como ocorre nas reações de HDS.

Argila: A remoção dos compostos sulfurados e nitrogenados obtida para cada resina avaliada á apresentada na Tabela 3. 
Tabela 3 - Resultados obtidos para a argila avaliada

\begin{tabular}{|c|c|c|c|c|}
\hline Adsorvente & $\begin{array}{c}\mathrm{q} \mathrm{S} \\
(\mathrm{mg} \mathrm{S} / \mathrm{g})\end{array}$ & $\begin{array}{c}\% \mathrm{~S} \\
\text { Removida }\end{array}$ & $\begin{array}{c}\mathrm{q} \mathrm{N} \\
(\mathrm{mg} \mathrm{N} / \mathrm{g})\end{array}$ & $\begin{array}{c}\% \mathrm{~N} \\
\text { Removida }\end{array}$ \\
\hline Argila & 0,6 & 26,6 & 0,8 & 39,9 \\
\hline
\end{tabular}

A argila Cleytone 40 removeu aproximadamente $27 \%$ dos compostos de enxofre e $40 \%$ dos compostos nitrogenados presentes no óleo diesel, resultados que também demonstram a maior afinidade dos compostos nitrogenados em relação aos sulfurados, como verificado para a maioria dos adsorventes testados. Santos (2011) avaliou a argila bentonítica Tonsil CO620 G na adsorção de compostos sulfurados e nitrogenados do óleo diesel hidrotratado, além de testar a argila pura (TCO), como recebida do fornecedor, testou também argilas impregnadas com níquel (TCO-Ni) e molibdênio (TCO-Mo). O autor verificou maior porcentagem de remoção para os compostos nitrogenados do que para os sulfurados pela amostra sem modificação e que ocorreu uma pequena melhora para TCO-Ni, e piora para TCO-Mo.

Carvão Ativado: A remoção dos compostos sulfurados e nitrogenados obtida para cada resina avaliada á apresentada na Tabela 4.

Tabela 4 - Resultados obtidos para a argila avaliada

\begin{tabular}{|c|c|c|c|c|}
\hline Adsorvente & $\begin{array}{c}\mathrm{q} \mathrm{S} \\
(\mathrm{mg} \mathrm{S} / \mathrm{g})\end{array}$ & $\begin{array}{c}\% \mathrm{~S} \\
\text { Removida }\end{array}$ & $\begin{array}{c}\mathrm{q} \mathrm{N} \\
(\mathrm{mg} \mathrm{N} / \mathrm{g})\end{array}$ & $\begin{array}{c}\% \mathrm{~N} \\
\text { Removida }\end{array}$ \\
\hline CA & 1,0 & 46,1 & 1,7 & 89,6 \\
\hline
\end{tabular}

De acordo com os resultados pode-se verificar que o carvão ativado, dentre os adsorventes avaliados, foi o que apresentou resultados mais satisfatórios quanto à remoção de compostos de enxofre e nitrogênio presentes no óleo diesel comercial.

Frente a este resultado novos estudos como avaliação do tempo de equilíbrio e obtenção de isotermas foram realizados apenas para o carvão ativado.

\subsection{Cinética de Adsorção}

As curvas obtidas para o tempo de equilíbrio para os compostos sulfurados e nitrogenados são apresentados na Figura 1, na qual se pode avaliar que após 4 horas de contato o ganho na remoção dos compostos de enxofre é de apenas $7 \%$ e para os compostos de nitrogênio é de $2 \%$, ganho que não justifica o gasto energético de mais 20 horas de processo. Com estes dados, estabeleceu-se o tempo de contato de 4 horas para o processo. 


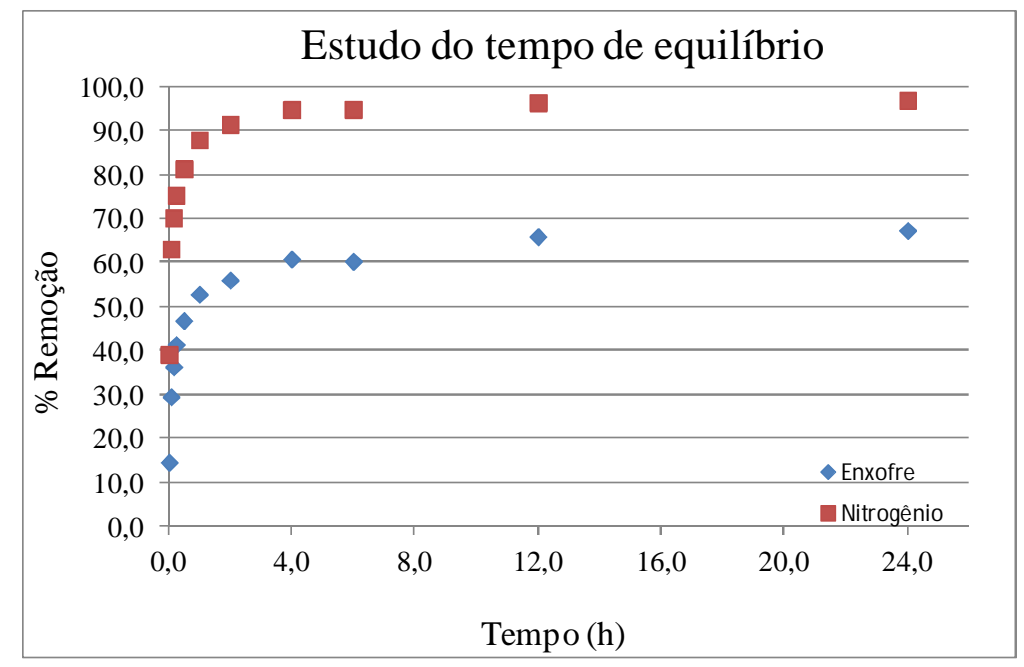

Figura 1 - Estudo do tempo de equilíbrio.

A partir dos resultados experimentais da quantidade adsorvida no tempo $\mathrm{t}$ em função do tempo, pode-se realizar a regressão dos dados pelas Equações 2 e 3, e assim calcularam-se os valores das constantes de velocidade $\mathrm{k}_{\mathrm{f}}$ e $\mathrm{k}_{\mathrm{s}}$, e de $\mathrm{q}_{\mathrm{e}}$ para o enxofre e nitrogênio, como apresentados nas Tabelas 5 e 6 respectivamente. $O$ valor obtido de $q_{e}$ para o enxofre foi de $0,85 \mathrm{mgS}_{\mathrm{g}} \mathrm{g}^{-1}$ e para o nitrogênio $\mathrm{q}_{\mathrm{e}}$ foi de $0,90 \mathrm{mgN} \cdot \mathrm{g}^{-1}$.

Tabela 5 - Resultados da análise cinética dos dois modelos avaliados para o enxofre

\begin{tabular}{|c|c|c|}
\hline \multicolumn{3}{|c|}{ Pseudo-Primeira Ordem } \\
\hline $\mathrm{q}_{\mathrm{e}, \text { cal }}\left(\mathrm{mg} \cdot \mathrm{g}^{-1}\right)$ & $\mathrm{k}_{\mathrm{f}}\left(\mathrm{min}^{-1}\right)$ & $\begin{array}{c}\text { Coeficiente } \\
\text { de Correlação }\left(\mathrm{R}^{2}\right)\end{array}$ \\
\hline 0,32 & 0,0007 & 0,8183 \\
\hline \multicolumn{3}{|c|}{ Pseudo-Segunda Ordem } \\
\hline $\mathrm{q}_{\mathrm{e}, \text { cal }}\left(\mathrm{mg} \cdot \mathrm{g}^{-1}\right)$ & $\mathrm{k}_{\mathrm{s}}\left(\mathrm{g} \cdot \mathrm{mg}^{-1} \cdot \mathrm{min}^{-1}\right)$ & $\begin{array}{c}\text { Coeficiente } \\
\text { de Correlação }\left(\mathrm{R}^{2}\right)\end{array}$ \\
\hline 0,84 & 0,0254 & 0,9994 \\
\hline
\end{tabular}

Tabela 6 - Resultados da análise cinética dos dois modelos avaliados para o nitrogênio

\begin{tabular}{|c|c|c|}
\hline \multicolumn{3}{|c|}{ Pseudo-Primeira Ordem } \\
\hline $\mathrm{q}_{\mathrm{e}, \text { cal }}\left(\mathrm{mg} \cdot \mathrm{g}^{-1}\right)$ & $\mathrm{k}_{\mathrm{f}}\left(\mathrm{min}^{-1}\right)$ & $\begin{array}{c}\text { Coeficiente } \\
\text { de Correlação }\left(\mathrm{R}^{2}\right)\end{array}$ \\
\hline 0,13 & 0,0006 & 0,4508 \\
\hline \multicolumn{3}{|c|}{ Pseudo-Segunda Ordem } \\
\hline $\mathrm{q}_{\mathrm{e}, \mathrm{cal}}\left(\mathrm{mg} \cdot \mathrm{g}^{-1}\right)$ & $\mathrm{k}_{\mathrm{s}}\left(\mathrm{g} \cdot \mathrm{mg}^{-1} \cdot \mathrm{min}^{-1}\right)$ & $\begin{array}{c}\text { Coeficiente } \\
\text { de Correlação }\left(\mathrm{R}^{2}\right)\end{array}$ \\
\hline 0,87 & 0,3870 & 0,9998 \\
\hline
\end{tabular}




\section{9 a 22 de outubro de 2014 \\ Florianópolis/SC}

Com tais resultados verificou-se que tanto para os compostos de enxofre quanto para os compostos de nitrogênio o modelo de pseudo-segunda ordem ajusta melhor os dados experimentais obtidos. Segundo Xu et al. (2013) esses resultados levam a conclusão de que a cinética de dessulfurização e desnitrogenação do óleo diesel utilizando carvão ativado como adsorvente nestas condições pode ser bem descrita pelo modelo de pseudo-segunda ordem.

\section{CONCLUSÃO}

Os adsorventes comerciais avaliados: resina, argila e carvão ativado apresentaram bons resultados na remoção dos compostos de enxofre e nitrogênio presentes no óleo diesel, sendo que dentre eles o carvão ativado se destacou, apresentando $46 \%$ de remoção dos compostos sulfurados e 90\% dos compostos nitrogenados.

Para o carvão ativado determinou-se que os dados foram melhor ajustados pelo modelo de pseudo-segunda ordem de Lagergren. Avaliou-se que o tempo de 4 horas de adsorção forneceu remoção de enxofre e nitrogênio satisfatória, não havendo a necessidade de prolongar o processo adsortivo.

Por fim, foi verificado que a técnica de adsorção com carvão ativado apresentou resultados promissores no que se refere à adsorção de compostos de enxofre e nitrogênio presentes no óleo diesel e que a continuidade dos estudos provavelmente acarretará em resultados ainda mais satisfatórios.

\section{REFERÊNCIAS}

AL-GHOUTIA, M.A.; AL-DEGSB, Y.S.; KHALILI, F.I. Minimization of organosulphur compounds by activated carbon from commercial diesel fuel: Mechanistic study. Chem. Eng. J., v. 162, p. 669-676, 2010.

BLANCO-BRIEVA, G; CAMPOS-MARTIN,J. M.; AL-ZAHRANI, S.M.; J.L.G. FIERRO, J. L.G. Effectiveness of metal-organic frameworks for removal of refractory organosulfur compound present in liquid fuels. Fuel, v. 90, p. 190-197, 2011.

FALLAH, R.; AZIZIAN, S. Removal of thiophenic compounds from liquid fuel by different modified activated carbon cloths. Fuel Process. Technol., v. 93, p. 45-52. 2012.

GUO, B.; WANG, R.; LI, Y. The performance of solid phosphoric acid catalysts and macroporous sulfonic resins on gasoline alkylation desulfurization. Fuel Process. Technol., v. 91, p. 1731-1735, 2010.

GUTIÉRREZ, O. Y., KLIMOVA, T. Effect of the support on high activity of the (Ni) o/ZrO2SBA-15 catalyst in the simultaneous hydrodesulfurization of DBT and 4,6-DMDBT. J. Catal., v. 281, p. 50-62, 2011.

KORIAKIN, A.; PONVEL, K. M.; LEE, C-H. Denitrogenation of raw diesel fuel by lithiummodified mesoporous silica. Chem. Eng. J., 162, 649-655, 2010. 
KUMAR, S.; SRIVASTAVA, V. C.; BADONI, R. P. Oxidative desulfurization by chromium promoted sulfated zirconia. Fuel Process. Technol., v. 93, p. 18-25, 2012.

PAZ-ZAVALA, C.; BURGOS-VÁZQUEZ, E.; RODRÍGUEZ-RODRÍGUEZ, J. E.; RAMÍREZVERDUZCO, L. F. Ultra low sulfur diesel simulation. Application to commercial units. Fuel: v. 110, p. 227-234. 2013.

SANTOS, A. L. Remoção de compostos sulfurados e nitrogenados de diesel hidrotratado por adsorção em argila, alumina e sílica-alumina, impregnadas com metais de transição. Dissertação de mestrado. Universidade do Estado do Rio de Janeiro. 2011.

TANABE, K. K.; COHEN, S. M. Postsynthetic modification of metal-organic frameworks-a progress report. Chem. Soc. Rev., v. 40, p. 498-519, 2011.

XIAO, J.; LI, Z.; LIU, B.; XIA, Q.; YU, M. Adsorption of Benzothiophene and Dibenzothiphene on Ion-Impregnated Activated Carbons and Ion-Exchanged Y Zeolites. Energ. Fuels, v. 22, p. 3858-3863, 2008.

XIE, L.; FAVRE-REGUILLON, A. Selective Extraction of Neutral Nitrogen-Containing Compounds from Straight-Run Diesel Feed Using Polymer-Supported Ionic Liquid Moieties. Ind. Eng. Chem. Res., v. 48, p. 3973-3977. 2009.

XIE, L.; FAVRE-REGUILLON, A.; WANG, X.; FU, X.; LEMAIRE, M. Selective Adsorption of Neutral Nitrogen Compounds from Fuel Using Ion-Exchange Resins. J. Chem. Eng. Data, v. 55, p. 4849-4853. 2010.

XU, X.; ZHANG, S.; LI, P.; SHEN, Y. Equilibrium and kinetics of Jet-A fuel desulfurization by selective adsorption at room temperatures. Fuel, v. 111, p. 172-179, 2013.

YANG, L.; WANG, S.; WANG, R.; YU, H.Selective Removal of Nitrogen-Containing Heterocyclic Compounds from Transportation Diesel Fuels with Reactive Adsorbent. Chin. J. Chem. Eng., v.21(5), p. 558-563, 2013. 